\title{
Towards IMIA 2015 - the IMIA Strategic Plan
}

\author{
Nancy M. Lorenzi, (IMIA President 2004-2007)
}

Vanderbilt University Medical Center, Nashville, TN, USA

\begin{abstract}
Summary
Objective: To report about the IMIA Strategic Plan 'Towards IMIA $2015^{\prime}$.

Method: Starting in 2004 with a survey of member needs, expectations and wishes, an IMIA task force elaborated this plan. It has been updated by IMIA General Assembly members in 2005 and 2006.

Results: A Conceptual Framework for IMIA's strategic plan has been elaborated. The IMIA Strategic Planning Framework stresses the following: (1) IMIA aims to improve biomedical research, clinical practice and publichealth (VISION); (2) IMIA aims to support investigation and development of advanced information systems and technologies (RESEARCH); (3) IMIA aims that its efforts are carried out in accordance with strict ethical and legal rules (BEHAVIORAL RESPONSIBILITY); (4) IMIA aims to promote education for and about biomedical informatics (EDUCATION); (5) IMIA aims to bridge relevant internal and external groups and organizations (RELATIONSHIP); (6) IMIA aims to incorporate multiple individuals, groups and organizations to constitute the IMIA Association. (REACH).

Conclusions: IMIA plays a major global role in the application of information science and technology in the fields of healthcare and research in medical, health and bio informatics. This framework provides IMIA with an excellent opportunity to focus its plans to ensure the highest probability of success is possible.

\section{Keywords}

Medical informatics, health informatics, biomedical informatics, strategic planning, International Medical Informatics Association, IMIA

Geissbuhler A, Haux R, KulikowskiC, editors. IMIA Yearbook of Medical Informatics 2007. Methods Inf Med 2007; 46Suppl 1: 1-5
\end{abstract}

2007 is a major year for the International Medical Informatics Association. This is the $40^{\text {th }}$ anniversary of our creation. We celebrate the year with our triennial Medinfo conference in Brisbane, Queensland, Australia. Also, we celebrate the year with the release of our strategic plan, "Towards IMIA 2015".

The IMIA strategic planning process started in 2004 with a survey of member needs, expectations and wishes. The results of the membership survey were outlined in the editorial 'The IMIA Possibility Framework' [1].

After the Medinfo 2004 meeting an IMIA Strategic Planning Task Force was created. The membership include, Nancy M. Lorenzi, IMIA President and Task Force chair, USA national representative, Vanderbilt University, Nashville, Tennessee, USA; Peter J. Murray, UK national representative, Chair Open Source Working Group, Lincoln, UK; Lincoln de Assis Moura Jr., Latin America and Caribbean Region representative and later IMIA Treasurer and Board Member, São Paulo, Brazil; H. M. Goh, Asia Pacific Association for Medical Informatics, Secretary, Kuala Lumpur, Malaysia; Steven Huesing, IMIA Executive Director, Edmonton, Alberta, Canada; Fernando Martin-Sanchez, Spain national representative, Chair Informatics in Genomic Medicine Working Group, Madrid, Spain; Floyd Eisenberg, Siemens Medical Solutions representative, Malvern, Pennsylvania, USA; Heather Strachan, Chair Nursing Informatics Special Interest Group, Bridge of Weir, UK.

The Task Force members reported the results of the efforts in the editorial, Strat- egy in a Fishbowl: An Invitation to Determine the Shape of IMIA in 2015 [2]. They also both updated and solicited feedback from the IMIA General Assembly members in 2005 and 2006. The remainder of this report is a result of the cumulative planning efforts.

\section{The IMIA Vision}

There will be a world-wide systems approach for healthcare. Clinicians, researchers, patients and people in general will be supported by a system of information technology tools, processes and behaviors that make it easy to do the right thing, in the right way, at the right time to improve health care for all. This systems approach will incorporate and integrate research, clinical care and general public health. To achieve this vision it will require everyone being supported by informaticsbased information and communication systems and technologies.

IMIA will provide leadership and expertise to the multidisciplinary health focused community and policy makers to enable the transformation of healthcare in accord with the world-wide vision to improve the health of the world population. IMIA will fulfill its vision by:

- Being the scientific informatics association through which the world's knowledge leaders come together to effectively and efficiently create, assemble, integrate, synthesize or assimilate intellectual knowledge that is required worldwide to advance biomedical informatics in its role of 
improving health and healthcare.

- Being the informatics association that effectively and efficiently connects people and the nations of the world to be able to accomplish the above purpose.

Inherent in IMIA's role is to bring together, from a global perspective, scientists, researchers, users, vendors, developers, consultants and suppliers in an environment of cooperation and sharing to research and develop the concepts needed to support the organizations of the world. As an organization committed to promoting best practice in the use of information and communication technologies within biomedical informatics and in health and healthcare, IMIA will ensure that it uses and promotes best practice in its own use of technology as a transformational strategic asset.

\section{IMIA's Guiding Principles}

The principles are: (1) IMIA will help to ensure a holistic, systems approach to collaboration and networking about biomedical informatics to maximize impact world-wide. (2) IMIA will uphold an ethical approach to informatics systems as identified by IMIA's ethical principles. (3) IMIA will promote biomedical informatics based on evidence and best practice to ensure impact on health is high quality, safe, efficient and sustainable and that makes it easy to do the right thing, in the right way, at the right time world-wide.

\section{IMIA's Integrated Strategic Framework Model}

IMIA as an organization and through it members has numerous possibilities for integration and connections. The
Strategic Planning Task Force evolved a two part model. The first part is a concentric circle component that conceptualizes and demonstrates the potential vastness of IMIA's connections and integration possibilities. The second is a "sector" concept component that outlines the key subject focused areas.

\section{Concentric Circle Concept Component}

The center of the set of concentric circles starts with knowledge and it is represented as the central core from which all other layers within the concentric circle originate (Figure 1). All of IMIA's strategies, interactions and efforts emanate from this core knowledge circle. Knowledge means all the concepts, methods, education, and research, among others that underpin biomedical informatics directly and indirectly.

The second circle (from the knowledge core) represents the science layer. It is within this layer of the concentric circle that IMIA members have their connection and integration with the science and discovery of informatics.

The third circle represents the application layer of the scientific discoveries. Within this circle are applications that address the multiple questions and issues in informatics are created, disseminated, evaluated, and so on.
The fourth circle represents IMIA's impact layer. This refers to the potential impact that IMIA and its members can have on governments, nations, outcomes, health professionals, individuals and others.

The fifth (or outer) circle represents the people layer. Items in this circle include individuals, citizen organizations, personal health involvement, dissemination and acceptance, enabling personal responsibility, and public/personal health.

\section{"Sector" Concept Component}

Another planning dimension outlines key subject-focused sectors. Superimposed on the five concentric circle layers are six major sectors. Knowledge is also the core of each of the six sectors. The doted lines between sectors mean that sectors interact among themselves. Although difficult to represent graphically, all sector are neighbors (Figure 2). The sectors start at the top of the circle with the health sector. The components within the health sector represent the IMIA vision. The components are: pathophysiology (science layer); diagnosis, therapy, treatment (application layer); public health and health improvement (impact layer) and personal health (people layer).
Fig. 1 The Five Concentric Circles of the IMIA Integrated Strategic Planning Framework

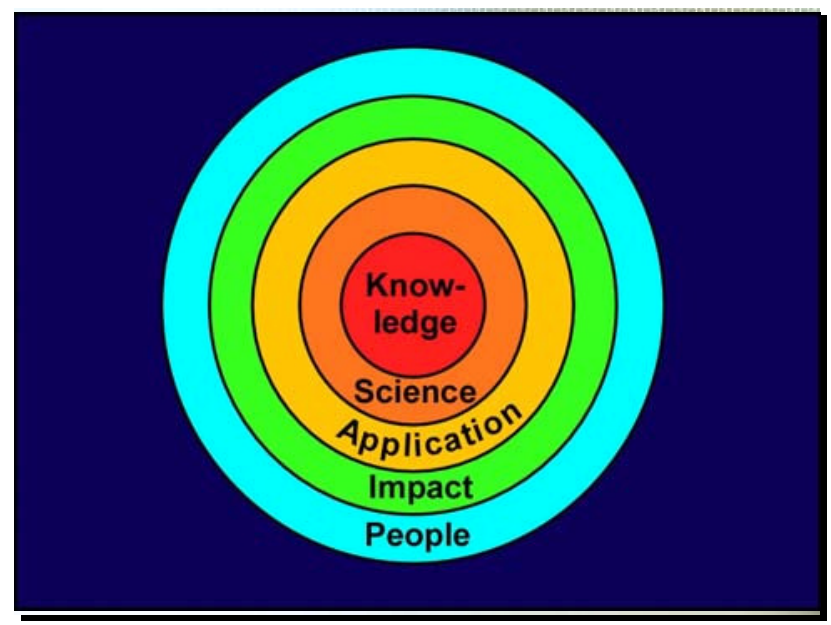


Fig. 2 The Six Sectors of the IMIA Integrated Strategic Planning Framework

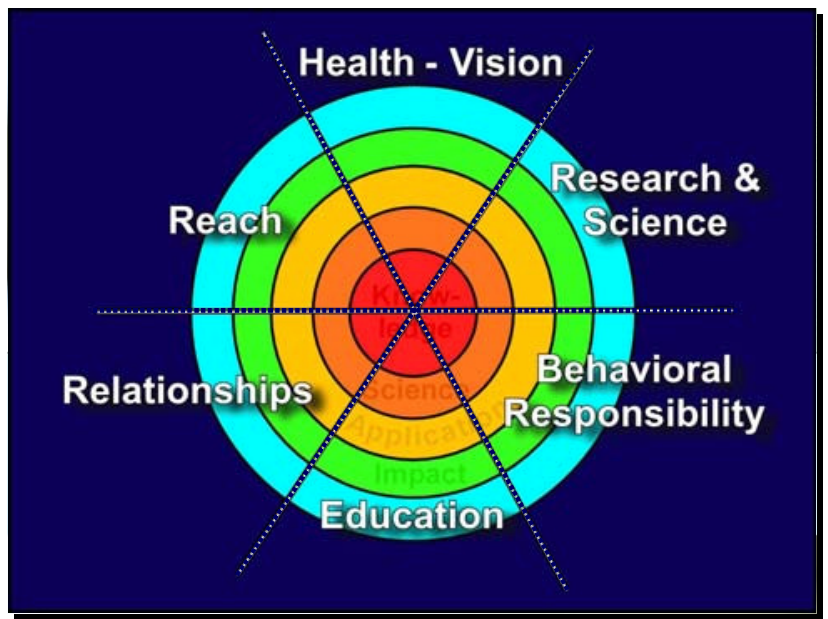

The next sector is research (how we understand and create to support health). The components within this sector include: basic core research (science layer); applied science (application layer); evaluation (usability) and outcomes (impact layer) and dissemination and acceptance (people layer). This is followed by the behavioral responsibility (ethics) sector (our ethical and social responsibility). Components within this sector include: ethics (science layer); legal/regulation (application layer); socio-economic (impact layer) and personal responsibility (people layer)

The next sector is education (best practices) (educating ourselves and others). This sector unfolds as: health informaticians (science layer); implementers (application layer); health professionals (impact layer) and people education (people layer)

The next sector represents the multiple types of relationships (communications and connections (our responsibility to build relationships among stakeholders). Within this sector are the following: academic (science layer); professional (application layer); governments/ agencies (impact layer); and citizen organizations (people layer)

The final sector is the reach (equity) of IMIA (our obligation to share, distrib- ute and disseminate). The components within this sector include: global (science layer); regions (application layer); nations (impact layer); and individuals (people layer).

\section{Brief Description of the Six "Pie Shaped" Sectors}

\section{Health}

The most important mandate for IMIA is to contribute, through the use of Information and Communication Technology (ICT), to the improvement of biomedical research, clinical practice and public health. It is envisioned that, in the coming years, a more complete knowledge on the different factors that contribute to the development of disease (genetics, environment) will be increasingly available for developing new preventive, diagnostic and therapeutic solutions. IMIA has a key role to play in anticipating new challenges for informatics in the most important trends of future medicine (regenerative, genomic, longevity, patient-centred, preventive). In this regard, working groups will have to demonstrate their leadership and provide guidance to the whole community.

\section{Research}

Biomedical informatics is a multi-disciplinary area that involves multiple content areas. It is one of the fastest growing subject/content areas in the world. The use of informatics is expected to enhance research efforts in areas such as genomics and proteomics, for example, and also to change the way medicine is practiced in the $21 \mathrm{st}$ century. Research in informatics ranges from the theoretical to applied efforts. The demand for more research in biomedical informatics and for biomedical informatics to support other researchers escalates daily. Knowledge is the core for IMIA's existence.

\section{Behavioral Responsibility}

IMIA has a responsibility to ensure that it develops, utilizes and shares the knowledge that it possesses responsibly. IMIA has a responsibility to ensure its members and through them the health of citizens of the world, benefit from this knowledge. Appropriate behavioral responsibility can support nations; organizations, communities and individuals get the right health information in the right format, to the right person, at the right time. The core science of IMIA's behavioral responsibility is the underlying approach to its activities, which is based on ethical rights and duties. IMIA promotes these rights and duties through its health informatics ethics principles including privacy and disposition, openness, security, access, legitimate infringement, least intrusive alternative and accountability.

\section{Education}

IMIA's raison d'etre is knowledge and education is the major means of knowledge transfer. IMIA has numerous roles 
to play in providing leadership in the development and delivery of education, and where appropriate through collaborating with other organizations in contributing to the development of education for biomedical informaticians, implementers, health professionals, and individual citizens. In this context, the word 'education' is used for all forms of education, training and professional development, although the precise mix of each, and the role that IMIA plays, will vary according to the group or content.

\section{Relationships}

IMIA has a responsibility to ensure productive and progressive growth in global health by building relationships among the various stakeholders. Biomedical informatics is a dynamic field requiring continuous interaction among various interests. Basic science research and education are essential to any field. However, application is required to improve global health by effecting workflow, communication, security and utility. Effective bi-directional relationships can support collaboration and true applicability of biomedical informatics. IMIA is the substrate to encourage growth and interaction among academic, professional, corporate, governmental and community-based citizen organizations.

\section{Reach}

IMIA believes that its members have a duty and obligation to share, distribute and disseminate knowledge to the world through its infrastructure and membership - in order to reach the ultimate beneficiary of the application of knowledge, the individual citizen.

\section{Strategic Goals}

The Strategic Planning Task Force outlined draft goals under each of the sectors. We are presenting the initial goals in this President's page, but these goals are currently being evaluated by people who were not on the task force and most likely they will be changed.

\section{Health Sector Goals}

The Health sector of the IMIA strategic plan represents the following:

- Science layer $=$ patho-physiology

- Application layer = diagnosis, therapy and treatment

- Impact layer $=$ public health, health improvement

- People layer $=$ personal

The following initial goals were developed to support this sector.

1. To develop a list of new challenges [data model created from the "omics"].

2. To outline what personalized medicine may mean for informatics, including the application and needs on a global level.

3. To develop teams with multiple national efforts to clarify the needs and roles of personal health records.

4. To develop a white paper that outlines strategies for an easy to use, widely available bio-surveillance strategy.

\section{Research Sector Goals}

The Research and Science sector represents:

- Science layer $=$ basic core research

- Application layer = applied science/ research

- Impact layer = evaluation (usability) and outcomes

- People layer $=$ very wide dissemination and acceptance

The following initial goals were developed to support this sector.
1. To establish international collaboration opportunities to network and to perform research worldwide.

2. Dissemination and acceptance of publications, e.g. Yearbook and journals, Medinfo, conferences, Working Groups.

3. To establish global connections to facilitate and improve informatics research methods.

4. To facilitate the global use of approved standards.

\section{Behavioral Responsibility Sector Goals}

The Behavioral Responsibility sector represents:

- Science layer $=$ ethics

- Application layer $=$ legal/regulation

- Impact layer = socio-economic

- People layer $=$ personal responsibility

The following initial goals were developed to support this sector.

1. To develop a comprehensive database of ethics best practice examples. Include both strategies and people for future contracts about ethical principles.

2. To develop a white paper regarding information governance worldwide. - White paper / recommendations.

3 . To work with consumers from multiple countries to clearly outline the behavioral responsibilities for the informaticians.

\section{Education Sector Goals}

This sector addresses;

- Science layer = health informaticians

- Application layer $=$ implementers

- Impact layer $=$ health professionals

- People layer $=$ people

The following initial goals were developed to support this sector.

1. To create models for potential informatics educational curricula based on the IMIA Knowledge Map and 
Competencies Map.

2. To map informatics competencies based on the IMIA Knowledge Map.

3. To endorse the certification process of others bases on the IMA Knowledge Map and Competencies Map.

4. To foster world-wide education collaboration of all IMIA members.

5. To foster informatics-based educational careers world-wide.

6. To provide information about curricula, competencies, certification and careers to IMIA members world-wide.

7. To continuously identify and address challenges to the informatics educational process.

\section{Relationship Sector Goals}

The Relationship sector represents:

- Science layer $=$ academic

- Application layer = professional

- Impact layer = governments/agencies

- People layer $=$ citizen organizations The following initial goals were developed to support this sector.

1. To facilitate connections among academic members for exchanges of ideas and people.

2. To facilitate connections with professional organizations (e.g. IFIP, IFHRO, AHIMA, Telehealth, HIMSS, Quality, among others) to further enhance global informatics efforts.

3. To facilitate connections with corporations to further enhance global informatics efforts.

4. To facilitate connections with the World Health Organization to further enhance global informatics efforts.

\section{IMIA as an Organization (Reach) Sector-Goals}

This sector addresses the following;

- Science layer = working groups
- Application layer $=$ nations

- Impact layer $=$ regions

- People layer = individuals/global

The following initial goals were developed to support this sector.

1. To facilitate and encourage communities of practice and interest worldwide.

2. To create the most effective communication strategies.

3. To develop strategies for conferences and meetings that meets the needs of the total IMIA family.

4. IMIA will develop a strategy to rapidly and effectively distribute and disseminate the materials that are created and produced or deemed important to IMIA members.

5. To achieve our strategic goals IMIA needs a financial strategy to fund the plan.

6. To meet its strategic goals IMIA requires an effective headquarters and governance strategy to lead and support its strategic goals.

7. To create an effective recognition program for the entire IMIA family whether individuals, member organizations or programs.

8. To create the most effective strategies to increase IMIA's visibility world-wide.

\section{Summary}

The IMIA Strategic Planning Framework stresses the following:

- IMIA aims to improve biomedical research, clinical practice and public health (VISION)

- IMIA aims to support investigation and development of advanced information systems and technologies (RESEARCH)

- IMIA aims that its efforts are carried out in accordance with strict ethical and legal rules (BEHAVIORAL RESPONSIBILITY)

- IMIA aims to promote education for and about biomedical informatics (EDUCATION)
- IMIA aims to bridge relevant internal and external groups and organizations (RELATIONSHIP)

- IMIA aims to incorporate multiple individuals, groups and organizations to constitute the IMIA Association. (REACH)

The Conceptual Framework for IMIA's strategic plan is comprehensive and demonstrates the multiple connections and interactions that are possible. This framework provides IMIA with an excellent opportunity to focus its plans to ensure the highest probability of success is possible.

IMIA is both a large organization and a small organization. It is large as it has many members that cover the world and it touches just about every aspect of informatics (whether it is biomedical, health medical, nursing or any other heading). It is small in that it has a small number of "staff" (the Executive director part time, the IMIA Board and the working group chairs). For all of us (IMIA plus all of you) to be successful we need to work together in a synergistic manner to create our future.

\section{References}

1. Lorenzi NM. The IMIA Possibility Framework. Methods Inf Med 2004;43:207.

2. Lorenzi NM, Murray PJ, de Assis Moura, Jr. L, Goh HM, Huesing S, Martin-Sanchez F, et al. Strategy in a fishbowl: an invitation to determine the shape of IMIA in 2015. Methods Inf Med 2006;45:235-9.

\section{Correspondence to:}

Nancy M. Lorenzi

Vanderbilt University Medical Center

Informatics Center, Eskind Biomedical Library

Biomedical Informatics

2209 Garland Avenue

Nashville, TN 37232-8340

USA

E-mail: nancy.lorenzi@vanderbilt.edu 